\title{
Validação de um instrumento multiprofissional para rastreio do risco de broncoaspiração em ambiente hospitalar
}

\author{
Validation of a multiprofessional instrument for screening the risk of bronchoaspiration in a \\ hospital environment \\ Validación de un instrumento multiprofesional para el cribado del riesgo de broncoaspiración en \\ un entorno hospitalario
}

\author{
Ana Paula de Andrade Sartori \\ ORCID: https://orcid.org/0000-0002-9633-7905 \\ Universidade Tuiuti do Paraná, Brasil \\ E-mail: anapaulafono10@gmail.com \\ Paulo Henrique Coltro \\ ORCID: https://orcid.org/0000-0002-0548-3561 \\ Universidade Tuiuti do Paraná, Brasil \\ E-mail: paulo_coltro@hotmail.com \\ Tatiane Caroline Boumer \\ ORCID: https://orcid.org/0000-0001-8538-5410 \\ Pontifícia Universidade Católica do Paraná, Brasil \\ E-mail: tatianeboumer@gmail.com \\ Isabel de Lima Zanata \\ ORCID: https://orcid.org/0000-0002-0902-5584 \\ Universidade Tuiuti do Paraná, Brasil \\ E-mail: isabelzanata@hotmail.com \\ Rosane Sampaio Santos \\ ORCID: https://orcid.org/0000-0001-6400-5706 \\ Universidade Tuiuti do Paraná, Brasil \\ E-mail: rosane.santos2@utp.br
}

\begin{abstract}
Resumo
Objetivo: Desenvolver e validar em conteúdo um instrumento multiprofissional para rastreio do risco de broncoaspiração. Método: Trata-se de um estudo transversal, quantitativo de validação de conteúdo que seguiu as seguintes etapas: elaboração de um instrumento de rastreio do risco de broncoaspiração com base em uma revisão bibliográfica e validação desse instrumento de acordo com concordância e relavância entre juízes. Para esta fase, 6 profissionais especialistas na area foram convidados ( 1 médico, 1 fisioterapeuta, 1 fonoaudiólogo, e 3 enfermeiros) a avaliar o instrumento e informar concordância baseadas em um questionário do tipo Likert. Para análise de concordância entre os juízes foi utilizado o Indice de Validade de Conteúdo para cada item (IVC-I) e total (IVC-T). Resultados: $\mathrm{O}$ instrumento foi composto por 10 itens: idade ( $\geq 60$ anos); doença pregressa; Escala de Glasgow $<13$; intubação orotraqueal; tempo de intubação orotraqueal ( $\geq 24 \mathrm{~h})$; traqueostomia; sensação de dispneia, higiêne oral inadequada; via alternativa de alimentação e tosse/engasgo. Há concordância entre juízes com relação a cada item (IVC-I 1,0) e também na totalidade (IVC-T 1,0) do instrumento. Conclusão: O instrumento multiprofissional para rastreio do risco de broncoaspiração em ambiente hospitalar foi desenvolvido e apresenta validade de conteúdo, assegurado por uma equipe de profissionais experientes na área.
\end{abstract}

Palavras-chave: Estudos de validação; Transtornos de deglutição; Programas de rastreamento; Broncopneumonia.

\footnotetext{
Abstract

Objective: To develop and content-validate a multidisciplinary instrument for screening the risk of bronchoaspiration. Method: This is a cross-sectional, quantitative content validation study that followed the following steps: development of an instrument for screening the risk of bronchoaspiration based on a literature review and validation of this instrument according to agreement and relevance among judges. For this phase, 6 specialists in the field were invited (1 physician, 1 physiotherapist, 1 speech therapist, and 3 nurses) to evaluate the instrument and report agreement based on a Likert-type questionnaire. For the analysis of agreement between the judges, the Content Validity Index was used for each item (CVI-I) and total (CVI-T). Results: The instrument consisted of 10 items: age ( $\geq 60$ years); past illness; Glasgow scale $<13$; orotracheal intubation; orotracheal intubation time ( $\geq 24$ hours); tracheostomy; feeling dyspneia,
} 
inadequate oral hygiene; via alternative feeding and coughing/choking. There is agreement between judges regarding each item (CVI-I 1.0) and also in the entirety (CVI-T 1.0) of the instrument. Conclusion: The multiprofessional instrument for screening the risk of bronchoaspiration in the hospital environment was developed and has content validity, provided by a team of experienced professionals in the field.

Keywords: Validation study; Deglutition disorders; Mass screening; Bronchopneumonia.

\section{Resumen}

Objetivo: Desarrollar y validar el contenido de un instrumento multidisciplinario para el cribado del riesgo de broncoaspiración. Método: Se trata de un estudio de validación de contenido cuantitativo, transversal, que siguió los siguientes pasos: desarrollo de un instrumento de cribado del riesgo de broncoaspiración a partir de la revisión de la literatura y la validación de este instrumento según concordancia y relevancia entre los jueces. Para esta fase se invitó a 6 especialistas en la materia ( 1 médico, 1 fisioterapeuta, 1 logopeda y 3 enfermeras) para evaluar el instrumento y reportar concordancia en base a un cuestionario tipo Likert. Para el análisis de concordancia entre los jueces se utilizó el Índice de Validez de Contenido para cada ítem (IVC-I) y total (IVC-T). Resultados: El instrumento constaba de 10 ítems: edad ( $\geq 60$ años); enfermedad pasada; Escala de Glasgow <13; intubación orotraqueal; tiempo de intubación orotraqueal ( $\geq 24 \mathrm{~h}$ ); traqueotomía; sensación de disnea, higiene bucal inadecuada; mediante alimentación alternativa y toser / atragantarse. Existe acuerdo entre los jueces en cada ítem (IVC-I 1.0) y también en la totalidad (IVC-T 1.0) del instrumento. Conclusión: El instrumento multiprofesional de cribado del riesgo de broncoaspiración en el ámbito hospitalario fue desarrollado y tiene validez de contenido, proporcionado por un equipo de profesionales con experiencia en la materia.

Palabras clave: Estudio de validación; Trastornos de deglución; Tamizaje masivo; Bronconeumonía.

\section{Introdução}

$\mathrm{O}$ ato de engolir é um processo fisiológico ordenado, que transfere o material ingerido ou a saliva da boca ao estômago (Dedivitis et al., 2017), qualquer comprometimento neste trajeto chama-se disfagia orofaríngea. A disfagia ocorre devido à dificuldade nos estágios iniciais da deglutição ou a sensação de obstrução na passagem de alimentos e/ou líquidos da boca para o estômago (Cho et al., 2015). Pode ser causada devido à quadros agudos, neurológicos ou de alterações das estruturas envolvidas na deglutição e capaz de gerar complicações graves, como a broncoaspiração (Yeo \& Rosenthal, 2018).

A broncoaspiração ocorre quando o paciente aspira conteúdo da boca ou do estômago para a laringe e trato respiratório inferior do pulmão (Marik, 2001). Os sintomas clínicos para a broncoaspiração são: tosse, dispnéia, voz molhada, engasgos ao se alimentar e podem estar acompanhados de sinais indicativos de disfagia orofaríngea, como: distúrbio de mastigação, dificuldade de deglutição, escape oral e nasal, sialorréia, recusa alimentar ou a diminuição da dieta por via oral (Bassi, 2014; Chaves, 2014; GOLD, 2018; Loivos, 2009).

Essa atenção frente ao risco de broncoaspiração é importante, pois tem-se observado que, a broncoaspiração aumenta as taxas de morbidade e mortalidade, prolongam em média 5 a 9 dias o tempo de internamento dos pacientes e maiores são os custos hospitalares (Carmo et al., 2018).

Existem várias situações no paciente hospitalizado que aumentam o risco de broncoaspiração pois comprometem o desempenho da deglutição (Fernandes \& Oda, 2017), entre eles: maior propensão de desenvolver pneumonias (DiBardino \& Wunderink, 2015), alterações clínicas devido as exacerbações de doenças de base e/ou comorbidades que podem levar ao rebaixamento do grau de cognição, do nível de consciência, da capacidade de comunicação e do controle motor.

É de extrema relevância que o rastreio da disfagia orofaríngea seja uma prática dos profissionais de diversas áreas da saúde e para que os indivíduos sejam encaminhados precocemente para o profissional especializado (Clavé \& Shaker, 2015). A atuação multidisciplinar, a qual envolve enfermeiros, médicos, fisioterapeutas e fonoaudiólogos, pode produzir impactos significativos no rastreio de pacientes em risco (Lee Titsworth et al., 2013), como por exemplo, na diminuição na prevalência de pneumonias. Os profissionais dessa equipe podem realizar ações preventivas, cujo o objetivo seja a 
diminuição das ocorrências de broncoaspiração e a minimização dos riscos aos quais o paciente pode estar exposto (Maeda \& Akagi, 2014).

Diante disso, criar um instrumento que permita auxiliar a equipe multiprofissional no rastreio aos riscos de broncoaspiração parece ser uma medida importante. Entretanto, desafiadora, pois a literatura apresenta vários instrumentos de rastreio da disfagia, como o The Eating Assessment Tool (EAT-10) (Belafsky et al., 2008); Dysphagia Screening Tool (Weinhardt et al., 2008) e MetroHealth Dysphagia Screen (Schrock et al., 2011), porém, não existe uma padronização quanto aos itens relevantes na identificação do paciente hospitalizado com risco de broncoaspiração em ambiente hospitalar.

Desta forma, pretende-se elaborar um instrumento de rastreio dos riscos de broncoaspiração em ambiente hospitalar que permitam nortear a equipe multiprofissional nas tomadas de decisões assistenciais. Mas além da criação, é necessário validá-la entre profissionais especialistas na área. Sendo assim, o objetivo deste estudo é desenvolver e validar em conteúdo um instrumento de aplicação multiprofissional para rastrear o risco de broncoaspiração.

\section{Metodologia}

Trata-se de um estudo transversal, quantitativo e de validação de conteúdo (Pereira et al., 2018) aprovado pelo Comitê de Ética da Universidade Tuiuti do Paraná (número 2.967.349).

O processo de validação aconteceu em duas etapas: seleção dos itens para compor a primeira versão do instrumento multiprofissional para rastreio do risco de broncoaspiração em ambiente hospitalar e validação em conteúdo do instrumento.

A versão do instrumento foi elaborada a partir da pesquisa sobre instrumentos que avaliam o risco de broncoaspiração. A elaboração do protocolo deu-se com base na literatura nacional e internacional, impressa e online. As bases de dados online foram a Biblioteca Virtual em Saúde (BVS), que contém as fontes relacionadas às ciências da saúde em geral, LILACS (Base de dados da Literatura Latino-Americana em Ciências da Saúde), MEDLINE (Base de dados Comprehensive MedLine), Biblioteca Cochrane, CidSaúde, SCIELO e Clinikal Key. O detalhamento desta revisão é apresentado no Quadro 1. 
Quadro 1 - Itens comumento utilizados para avaliar risco de broncoaspiração com base na revisão de literatura.

\begin{tabular}{|c|c|}
\hline $\begin{array}{c}\text { Literatura } \\
\text { Autores e ano }\end{array}$ & Itens utilizados para avaliar risco de broncoaspiração \\
\hline Baijens et al. (2016); Payne e Morley (2017) & $\begin{array}{c}\text { Histórico de doença pregressa (neurológica, respiratória, } \\
\text { esofágica,gástrica, cirurgia de cabeça e pescoço) }\end{array}$ \\
\hline Dedivitis et al. (2017) & Escala de Glasgow <13 \\
\hline $\begin{array}{c}\text { Souza et al. (2016); Mendes (2018) Kwok et al. } \\
\text { (2013); Skoretz, Flowers e Martino (2010) }\end{array}$ & Intubação orotraqueal \\
\hline Dedivitis et al. (2017) & Tempo em intubação orotraqueal $\geq 24$ horas \\
\hline Dedivitis et al. (2017) & Traqueostomia \\
\hline Dedivitis et al. (2017) & Hignéia \\
\hline Dedivitis et al. (2017); Arruda (2018) & Toral adequada \\
\hline Nascimento (2018) & Via alternativa de alimentação (SNE, GTT, Jejunostomia) \\
\hline Toh Yoon (2018) & Turante a alimentação ou com saliva \\
\hline Fritz, Suiter e Postma (2019) & \\
\hline
\end{tabular}

Fonte: Autores (2021)

No Quadro 2, é apresentado o instrumento utilizado para rastreio dos riscos de broncoaspiração.

Quadro 2- Instrumento multiprofissional para rastreio do risco de broncoaspiração em ambiente hospitalar.

\section{Instrumento multiprofissional para rastreio do risco de broncoaspiração em ambiente hospitalar}

\begin{tabular}{|l|l|l|}
\hline \multicolumn{1}{|c|}{ Questões } & Sim (1) & Não (0) \\
\hline 1. Paciente possui $\geq 60$ anos? & & \\
\hline $\begin{array}{l}\text { 2. Apresenta histórico de doença pregressa (neurológica, respiratória, } \\
\text { esofágica, gástrica, cirurgia de cabeça e pescoço)? }\end{array}$ & & \\
\hline 3. Paciente apresenta Escala de Glasgow <13? & & \\
\hline 4. Necessitou de intubação orotraqueal? & & \\
\hline 5. Tempo em intubação orotraqueal $\geq 24$ horas: & & \\
\hline 6. Utiliza traqueostomia? & & \\
\hline 7. Paciente apresenta sensação de dispneia? & & \\
\hline 8. Paciente apresenta higiêne oral adequada? & & \\
\hline 9. Paciente utiliza via alternativa de alimentação (SNE, GTT, Jejunostomia)? & & \\
\hline 10. Paciente apresenta tosse/engasgo durante a alimentação ou com saliva? & & \\
\hline
\end{tabular}

A pontuação máxima para este instrumento é de 10 pontos, onde 0 indica que o paciente não tem risco de broncoaspiração e valores $\geq 1$ indicam paciente em risco de broncoaspiração. Fonte: Autores (2021).

Foram convidados 6 profissionais para participar da pesquisa que assinaram o termo de consentimento livre e esclarecido. Os profissionais foram selecionados seguindo o critério de inclusão: atuação em área hospitalar e vivência prática ao tema broncoaspiração. Foram convidados profissionais da região Sul e Sudeste do Brasil. Participaram do 
processo de validação 6 juízes sendo 1 médico, 1 fisioterapeuta, 1 fonoaudiólogo e 3 enfermeiros. As características da amostra estão apresentadas no Quadro 3.

Quadro 3 - Características da amostra de juízes que participaram do estudo.

\begin{tabular}{|c|l|l|c|c|}
\hline $\begin{array}{l}\text { Categoria } \\
\text { profissional }\end{array}$ & Gênero & $\begin{array}{l}\text { Máxima titulação } \\
\text { acadêmica }\end{array}$ & $\begin{array}{l}\text { Tempo de atuação } \\
\text { na área profissional }\end{array}$ & $\begin{array}{l}\text { Vivência relacionada ao } \\
\text { tema broncoaspiração }\end{array}$ \\
\hline Médico & Feminino & Mestrado & Mais de 20 anos & Prática \\
\hline Fisioterapeuta & Feminino & Mestrado & 11 a 20 anos & Prática e acadêmica \\
\hline Fonoaudiólogo & Feminino & Doutorado & 6 a 10 anos & Prática \\
\hline Enfermeiro & Masculino & Mestrado & 1 a 5 anos & Prática e acadêmica \\
\hline Enfermeiro & Feminino & Especialização & 6 a 10 anos & Prática \\
\hline Enfermeiro & Feminino & Especialização & & \\
\hline
\end{tabular}

Fonte: Autores (2021).

Foram questionados aos juízes a concordância em 10 itens através de perguntas e mais o somatório do instrumento. As respostas quanto à avaliação de concordância foram do tipo categóricas baseadas no questionário de Likert, onde os juizes poderiam responder: "não entendi", “entendi pouco", "entendi parcialmente”, "entendi plenamente".

Para a análise de concordância entre os juízes foi utilizado o Índice de Validade de Conteúdo para cada item (IVC-I) para cada item do instrumento (Cálculo 1).

$$
I V C I=\frac{\text { número de respostas concordantes }}{\text { número total de respostas }}
$$

onde,

IVCI, corresponde ao Índice de Validade de Conteúdo para cada item;

número de respostas concordantes, corresponde ao número de juízes que responderam ao item como "entendi plenamente" ou "entendi parcialmente";

número total de respostas, corresponde ao número total de respostas informadas pelos juizes.

Posteriormente à análise de concordância de cada item, foi realizado o Índice de Validade de Conteúdo Total (IVCT) do instrumento, esse cálculo foi realizado com base no somatório de todos os IVC-I, dividido pelo número de itens do instrumento.

O valor de IVC pode variar de 0 a 1.0, onde quanto mais próximo a 1.0 maior a concordância entre os juízes. Para este estudo, considerou o número de 6 juízes. Para essa quantidade, pode-se existir uma pequena não-concordância, ou seja, o valor mínimo para que o instrumento seja validado foi de 0.79 .

Além do questionamento aos juízes quanto a concordância, os mesmos foram questionados quanto à relevância de cada item do instrumento com relação ao risco de broncoaspiração. 
Research, Society and Development, v. 11, n. 1, e27111124847, 2022

(CC BY 4.0) | ISSN 2525-3409 | DOI: http://dx.doi.org/10.33448/rsd-v11i1.24847

\section{Resultados}

Com base nos resultados apresentados na Tabela 1, todos os juízes apresentaram concordância com relação a cada item do instrumento e também em sua totalidade.

Tabela 1 - Índices de validade de conteúdo do instrumento - análise dos juízes.

\begin{tabular}{llc}
\hline Itens & $\begin{array}{c}\text { Número de juízes que consideraram o item } \\
\text { "entendi plenamente" ou "entendi parcialmente" }\end{array}$ & IVC-I* \\
\hline Paciente possui $\geq 60$ anos? & 6 & 1 \\
Apresenta histórico de doença pregressa? & 6 & 1 \\
Paciente apresenta escala de Glasgow $<13 ?$ & 6 & 1 \\
Necessitou de intubação orotraqueal? & 6 & 1 \\
Tempo em intubação orotraqueal $\geq 24 h ?$ & 6 & 1 \\
Utiliza traqueostomia? & 6 & 1 \\
Paciente apresenta sensação de dispnéia? & 6 & 1 \\
Paciente apresenta higiene oral inadequada? & 6 & 1 \\
Paciente utiliza via alternativa de alimentação? & 6 & 1 \\
$\begin{array}{l}\text { Paciente apresenta tosse/engasgo durante a } \\
\text { alimentação ou com a saliva? } \\
\text { Somatório }\end{array}$ & 6 & 6 \\
\hline
\end{tabular}

Legenda: *Índice de Validade de Conteúdo do Instrumento (IVCI) e **índice de Validade de Conteúdo Total (IVCT). Fonte: Autores (2021).

Na Tabela 2, estão apresentadas as opiniões dos juízes com relação a relevância dos itens para avaliar o risco de broncoaspiração. 
Tabela 2 - Distribuição de frequências das opiniões dos juízes com relação à relevância dos itens para avaliar riscos de broncoaspiração.

\begin{tabular}{|c|c|c|c|c|}
\hline \multirow[b]{2}{*}{ Itens } & \multicolumn{4}{|c|}{ Relevância } \\
\hline & $\begin{array}{l}\text { Considero essa } \\
\text { questão muito } \\
\text { relevante } \\
\mathrm{n}(\%)\end{array}$ & $\begin{array}{l}\text { Considero essa } \\
\text { questão } \\
\text { parcialmente } \\
\text { relevante } \\
\text { n }(\%)\end{array}$ & $\begin{array}{l}\text { Considero } \\
\text { essa questão } \\
\text { pouco } \\
\text { relevante } \\
\text { n (\%) }\end{array}$ & $\begin{array}{c}\text { Não considero essa } \\
\text { questão relevante } \\
\mathrm{n}(\%)\end{array}$ \\
\hline Paciente possui $\geq 60$ anos? & $6(100 \%)$ & $0(0 \%)$ & $0(0 \%)$ & $0(0 \%)$ \\
\hline $\begin{array}{l}\text { Apresenta histórico de doença } \\
\text { pregressa? }\end{array}$ & $6(100 \%)$ & $0(0 \%)$ & $0(0 \%)$ & $0(0 \%)$ \\
\hline $\begin{array}{l}\text { Paciente apresenta escala de } \\
\text { Glasgow }<13 \text { ? }\end{array}$ & $5(83,3 \%)$ & $1(16,7 \%)$ & $0(0 \%)$ & $0(0 \%)$ \\
\hline $\begin{array}{l}\text { Necessitou de intubação } \\
\text { orotraqueal? }\end{array}$ & $5(83,3 \%)$ & $1(16,7 \%)$ & $0(0 \%)$ & $0(0 \%)$ \\
\hline $\begin{array}{l}\text { Tempo em intubação orotraqueal } \\
\geq 24 \mathrm{~h} \text { ? }\end{array}$ & $6(100 \%)$ & $0(0 \%)$ & $0(0 \%)$ & $0(0 \%)$ \\
\hline Utiliza traqueostomia? & $5(83,3 \%)$ & $1(16,7 \%)$ & $0(0 \%)$ & $0(0 \%)$ \\
\hline $\begin{array}{l}\text { Paciente apresenta sensação de } \\
\text { dispnéia? }\end{array}$ & $3(50 \%)$ & $2(33,3 \%)$ & $1(16,7 \%)$ & $0(0 \%)$ \\
\hline $\begin{array}{l}\text { Paciente apresenta higiene oral } \\
\text { inadequada? }\end{array}$ & $4(66,7 \%)$ & $1(16,7 \%)$ & $0(0 \%)$ & $1(16,7 \%)$ \\
\hline $\begin{array}{l}\text { Paciente utiliza via alternativa de } \\
\text { alimentação }\end{array}$ & $6(100 \%)$ & $0(0 \%)$ & $0(0 \%)$ & $0(0 \%)$ \\
\hline $\begin{array}{l}\text { Paciente apresenta tosse/engasgo } \\
\text { durante a alimentação ou com a } \\
\text { saliva? }\end{array}$ & $6(100 \%)$ & $0(0 \%)$ & $0(0 \%)$ & $0(0 \%)$ \\
\hline Somatório & $6(100 \%)$ & $0(0 \%)$ & $0(0 \%)$ & $0(0 \%)$ \\
\hline
\end{tabular}

Fonte: Autores (2021).

\section{Discussão}

Foi possível definir e validar os itens mais relevantes no rastreio do risco de broncoaspiração em ambiente hospitalar após análise dos juízes. O trabalho é significativo, pois na prática hospitalar cada serviço opta em descrever e considerar seus itens de risco de broncoaspiração conforme a demanda e população a qual atendem. Já o instrumento validado contempla a unificação dos itens às diferentes populações de pacientes hospitalizados.

As áreas escolhidas que compuseram o quadro de juízes (médico, enfermeiro, fisioterapeuta e fonoaudiólogo) foram consideradas por serem aquelas que, geralmente, apresentam o contato imediato com o paciente recém-internado, identificando os fatores de risco de broncoaspiração previamente. Além disso, são as áreas mais presentes em processos como intubação orotraqueal e extubação, detendo de conhecimentos mais específicos para analisarem de maneira mais criteriosa os itens do instrumento proposto para a validação nesta primeira etapa, ou seja, de conteúdo. Há um número maior de juízes com formação em Enfermagem, pois considerou-se o fato de serem os profissionais que estão 24 horas com o paciente e, por isso, teoricamente, podem aplicar o instrumento mais precocemente do que as demais areas envolvidas, identificando mais rapidamente os pacientes em risco de broncoaspiração.

Pessoas com 60 anos ou mais apresentam aumento da prevalência de fragilidade física e os riscos de declínio podem combinar para um impacto negativo da função da deglutição (Paine, 2017). No primeiro item do instrumento "Paciente possui $\geq 60$ anos?", houve concordância entre todos os juízes em validade e relevância. O critério converge com a 
literatura, pois Madhavan et al. (2018), em seus estudos, coloca o critério de inclusão pacientes > 60 anos já que há evidências no que se refere às mudanças na deglutição, nutrição e habilidades físicas a partir da sexta década de vida (Madhavan et al., 2018).

Não é raro estes pacientes idosos, ou não, apresentarem doenças pregressas que podem alterar o padrão de deglutição. O segundo item do instrumento contempla o critério, “Apresenta histórico de doença pregressa?”, validado pelos juízes. O critério validado corrobora com a literatura (Carmo et al., 2018) onde ao propor um gerenciamento do risco de broncoaspiração em pacientes com disfagia orofaríngea, comprovaram o predomínio de neuropatias como risco. Para Delegge (2002) além das doenças neurológicas outros fatores estão associados ao risco de broncoaspiração como cirurgias de cabeça e pescoço e doenças pulmonares (DeLegge, 2002), também, contemplados no item.

A Escala de Coma de Glasgow determina alterações do nível de consciência de forma global e, por isso, é um dos critérios usados na construção do instrumento: "Paciente apresenta escala de Glasgow < 13?". No estudo de Furmann e Costa (2015) ao identificar critérios clínicos utilizados por profissionais para liberação de alimentação via oral em pacientes hospitalizados, a Escala de Coma de Glasgow foi a escala mencionada pelos 45 profissionais participantes, no total de 48 (Furmann \& Costa, 2015). O item validado pelos juízes constata com Medeiros e seus colaboradores (2016) onde ao correlacionar a gravidade de pacientes críticos não neurológicos com preditores clínicos de broncoaspiração, concluíram no estudo como um dos preditores a Escala de Coma de Glasgow, com escore $\leq 14$ (Medeiros et al., 2016).

Do mesmo modo, o quinto, o sexto e o sétimo itens do instrumento, "Tempo de intubação $\geq 24$ horas?”, "Utiliza traqueostomia?" e "Paciente apresenta sensação de dispnéia?”, respectivamente, foram validados pelo grupo de juízes. Há concordância dos mesmos critérios na literatura mostrado em um estudo de Almeida et al., (2017) que considera esses fatores preditivos de risco para disfagia orofaríngea (Almeida et al., 2017).

A intubação orotraqueal causa alterações estruturais da anatomia da glote, edemas e interrupção do reflexo de deglutição. E, por isso, recomenda-se que os pacientes com mais de dois dias de ventilação mecânica, recebam avaliação clínica objetiva da deglutição (Dedivitis et al., 2017). Dado tamanha importância, é que a maioria dos juízes considerou o quarto, "Necessitou de intubação orotraqueal?", e o quinto itens, "Tempo em intubação orotraqueal $\geq 24$ horas?", muito relevantes ao risco de broncoaspiração e validaram ambos itens. Em um estudo de coorte retrospectivo, See e colaborados (2016) fizeram uma análise de grupos de pacientes extubados com > 72 horas de ventilação mecânica, considerando que maior tempo de intubação orotraqueal predispõe pacientes à disfagia pós extubação (See et al., 2016). Ainda, nos estudos de Nascimento e colaboradoradores (2018) e Oliveira e colaboradores (2018), o aumento do tempo de intubação orotraqueal elevou em 5,5 vezes o risco de aspiração (Nascimento et al., 2018; Oliveira et al., 2018). É uma prática comum ocorrer a avaliação da deglutição até 24 horas ou mais após extubação, com base no pressuposto de que a função da deglutição melhora nas primeiras 24 horas (Marvin et al., 2019).

O sétimo item, "Paciente apresenta sensação de dispnéia?", foi validado pelo grupo de juizes. A dispnéia, juntamente com a dor torácica e a tosse, configuram uma tríade sintomatológica clássica das pneumonias (Porto \& Porto, 2019).

A higiene bucal em pacientes enfermos previne infecções e doenças secundárias como pneumonia aspirativa (Nascimento et al., 2018). O oitavo item do instrumento, “Paciente apresenta higiene oral inadequada?”, foi validado pelos juízes. Convergindo com alguns estudos, pois Cavalcante e Matos (2015) constataram que a implantação da prática correta de higiene oral nos pacientes críticos, contribuiu com uma significativa redução de infecções em unidade de terapia intensiva (Cavalcante \& Matos, 2015) e Madhavan e seus colaboradores (2018), ao desenvolverem uma ferramenta de triagem para identificação da disfagia, incluíram como um dos norteadores a saúde bucal (Madhavan et al., 2018). 
Existem alguns eventos adversos que podem ocorrer relacionados com pneumonia aspirativa pelo uso de nutrição enteral como: refluxo gastroesofágico e intolerância alimentar, podendo haver volume residual gástrico (Toh Yoon, 2018). Sabe-se que alguns pacientes em internação fazem uso de via alternativa de alimentação, por consequente, o nono item, "Paciente utiliza via alternativa de alimentação?", foi utilizado como critério de risco de broncoaspiração e validado pelo grupo de juízes.

Em relação a traqueostomia, é sabido que o dispositivo provoca alterações neurofisiológica e mecânicas no processo de coordenação da respiração e deglutição, aumentando o risco de aspiração (Dedivitis et al., 2017). O uso da traqueostomia foi usado com critério do instrumento e validado pelos juízes em concordância e relevância.

Behera e colaboradores (2018) realizaram um estudo para desenvolver uma ferramenta de triagem de deglutição válida e confiável, em pacientes com Acidente Vascular Cerebral, e consideraram dentre os critérios norteadores: histórico de uso de via alternativa de alimentação e uso de traqueostomia (Behera et al., 2018).

Entende-se que a aspiração ou penetração de líquidos ou alimentos na laringe provoca reflexo de tosse (Fritz et al., 2019). No décimo item do instrumento, "Paciente apresenta tosselengasgo durante a alimentação ou com a saliva?", houve entre os juízes concordância total em relevância, o que corrobora com o estudo de Ferrucci e colaboradores (2019), que consideraram um dos sinais clínicos preditores de broncoaspiração a presença de tosse após deglutição (Ferrucci et al., 2019).

O item "Somatório" também foi validado pelos juízes. No caso de não haver risco de broncoaspiração, recomendase higienização oral e vigilância do paciente na presença de risco. Na presença de risco para o paciente, recomenda-se cabeceira do leito $\mathrm{em} \geq 30^{\circ}$, higienização oral e acionamento da equipe multidisciplinar. Intervenções recomendadas também pela NIC - Classification of Nursing Interventions, como posicionar o paciente verticalmente com ângulo igual ou superior a $30^{\circ}$, podendo atingir até $90^{\circ}$ se houver possibilidade; manter cabeça elevada 30 a 40 minutos após a alimentação e prestar cuidados bucais (Butcher et al., 2016).

A validação de conteúdo avalia o grau em que cada elemento de um instrumento de medida é relevante e representativo de um específico constructo com um propósito particular de avaliação, sendo fundamental no processo de desenvolvimento e adaptação de instrumento de medidas. Entretanto, sua utilização não elimina a necessidade de aplicação de outras medidas psicométricas adicionais. Efetuar todas as etapas é uma forma de contribuir para a construção de instrumentos clínicos, os quais possuem baixo custo e considerável relevância social, tendo ainda um elevado índice de evidências científicas.

\section{Conclusão}

O instrumento multiprofissional para rastreio do risco de broncoaspiração em ambiente hospitalar foi desenvolvido e apresenta validade de conteúdo, assegurado por uma equipe especialista na área.

Sugere-se a continuidade no processo de validação do instrumento multiprofissional para rastreio do risco de broncoaspiração em ambiente hospitalar, quanto a sua aplicabilidade por profissionais habilitados, envolvendo maior gama destes exemplos, nutricionistas, odontólogos e técnicos de enfermagem.

\section{Referências}

Almeida, T. M., Cola, P. C., Pernambuco, L. de A., Magalhães, H. V., Magnoni, C. D., \& Silva, R. G. da. (2017). Instrumento de rastreio para disfagia orofaríngea no Acidente Vascular Encefálico - Parte I: evidências de validade baseadas no conteúdo e nos processos de resposta. CoDAS, 29(4), 1-9. https://doi.org/10.1590/2317-1782/20172017009

Bassi, D. (2014). Doença pulmonar obstrutiva cônica: considerações sobre deglutição e qualidade de vida (Issues 1-112). UNIVERSIDADE FEDERAL 
Research, Society and Development, v. 11, n. 1, e27111124847, 2022

(CC BY 4.0) | ISSN 2525-3409 | DOI: http://dx.doi.org/10.33448/rsd-v11i1.24847

\section{DE SANTA CATARINA.}

Behera, A., Read, D., Jackson, N., Saour, B., Alshekhlee, D., \& Mosier, A. K. (2018). A Validated Swallow Screener for Dysphagia and Aspiration in Patients with Stroke. Journal of Stroke and Cerebrovascular Diseases, 27(7), 1897-1904. https://doi.org/10.1016/j.jstrokecerebrovasdis.2018.02.037

Belafsky, P. C., Mouadeb, D. A., Rees, C. J., Pryor, J. C., Postma, G. N., Allen, J., \& Leonard, R. J. (2008). Validity and reliability of the eating assessment tool (EAT-10). Annals of Otology, Rhinology and Laryngology, 117(12), 919-924. https://doi.org/10.1177/000348940811701210

Butcher, H. K., Bulechek, Gloria M. Dochterman, J., \& Wagner, C. M. (2016). NIC - Classificação das Intervenções de Enfermagem. Elsevier.

Carmo, L. F. dos S., Santos, F. A. A. dos, Mendonça, S. C. B. de, \& Araújo, B. C. L. (2018). Management of the risk of bronchoaspiration in patients with oropharyngeal dysphagia. Revista CEFAC, 20(4), 532-540. https://doi.org/10.1590/1982-021620182045818

Cavalcante, L. da S., \& Matos, M. do P. S. de O. (2015). Práticas de higienização oral ao paciente da UTI e efeitos benéficos na análise de 30 enfermeiros no Pronto Socorro e Hospital 28 de Agosto em Manaus/AM. J. Health Sci. Inst, 33(3), 239-242. https://www.unip.br/comunicacao/publicacoes/ics/edicoes/2015/03_jul-set/V33_n3_2015_p239a242.pdf

Chaves, R. de D. (2014). Achados clínicos e da análise videofluoroscópica da deglutição em pacientes com doença pulmonar obstrutiva crônica [Universidade de São Paulo]. https://www.teses.usp.br/teses/disponiveis/5/5162/tde-26052014-104709/pt-br.php

Cho, S. Y., Choung, R. S., Saito, Y. A., Schleck, C. D., Zinsmeister, A. R., Locke III, G. R., \& Talley, N. J. (2015). Prevalence and risk factors for dysphagia: a U.S. community study. Neurogastroenterol Motil, 27(2), 212-219. https://doi.org/10.1111/nmo.12467.

Clavé, P., \& Shaker, R. (2015). Dysphagia: Current reality and scope of the problem. Nature Reviews Gastroenterology and Hepatology, 12(5), 259-270. https://doi.org/10.1038/nrgastro.2015.49

Dedivitis, R. A., Santoro, P. P., \& Arakawa-Sugueno, L. (2017). Manual prático de disfagia - diagnóstico e tratamento (1 ${ }^{\mathrm{a}}$ edição). Reviter. https://doi.org/10.1017/CBO9781107415324.004

DeLegge, M. H. (2002). Aspiration Pneumonia: Incidence, Mortality, and At-Risk Populations. In Journal of Parenteral and Enteral Nutrition (Vol. 26, Issue 6_suppl, pp. S19-S25). https://doi.org/10.1177/014860710202600604

DiBardino, D. M., \& Wunderink, R. G. (2015). Aspiration pneumonia: A review of modern trends. Journal of Critical Care, 30(1), 40-48. https://doi.org/10.1016/j.jcrc.2014.07.011

Fernandes, K., \& Oda, S. (2017). Manual Prático de disfagia - diagnóstico e tratamento. (1ª).

Ferrucci, J. L., Sassi, F. C., de Medeiros, G. C., \& de Andrade, C. R. F. (2019). Comparison between the functional aspects of swallowing and clinical markers in ICU patients with Traumatic Brain Injury (TBI). Codas, 31(2), 1-11. https://doi.org/10.1590/2317-1782/20182017278

Fritz, M. A., Suiter, D. M. \& Postma, G. (2019). Chronic Cough. In Dysphagia in Chronic Cough. Plural Publishing, Inc.

Furmann, N., \& Costa, F. M. (2015). Critérios clínicos utilizados por profissionais para liberação de dieta via oral em pacientes adultos hospitalizados. Revista CEFAC, 17(4), 1278-1287. https://doi.org/10.1590/1982-0216201517413614

Global strategy for the diagnosis, managemente, and prevention of chronic obstructive pulmonary disease. GOLD, Global Obstructive Lung Disease, (2018), 1-44. http://www.goldcopd.org/uploads/users/files/GOLD_Report_2015_Apr2.pdf

Lee Titsworth, W., Abram, J., Fullerton, A., Hester, J., Guin, P., Waters, M. F., \& Mocco, J. (2013). Prospective quality initiative to maximize dysphagia screening reduces hospital-acquired pneumonia prevalence in patients with stroke. In Stroke (Vol. 44, Issue 11, pp. 3154-3160). https://doi.org/10.1161/STROKEAHA.111.000204

Loivos, L. (2009). DPOC - definição e conceitos - as bases clínicas. Pulmão RJ - Atualizações Temáticas, 1(1), 34-37. http://www.sopterj.com.br/atualizacoes_tematicas/2009/05.pdf

Madhavan, A., Carnaby, G. D., Chhabria, K., \& Crary, M. A. (2018). Preliminary development of a screening tool for pre-clinical dysphagia in community dwelling older adults. Geriatrics (Switzerland), 3(4), 1-12. https://doi.org/10.3390/geriatrics3040090

Maeda, K., \& Akagi, J. (2014). Oral care may reduce pneumonia in the tube-fed elderly: A preliminary study. Dysphagia, 29(5), 616-621. https://doi.org/10.1007/s00455-014-9553-6

Marik, P. (2001). Aspiration Syndromes. In Handbook of Evidence-Based Critical Care. Springer-Verlag. https://doi.org/10.1016/B978-0-323-01199$0.50216-4$

Marvin, S., Thibeault, S., \& Ehlenbach, W. J. (2019). Post-extubation Dysphagia: Does Timing of Evaluation Matter? In Dysphagia (Vol. 34, Issue 2, pp. 210-219). https://doi.org/10.1007/s00455-018-9926-3

Medeiros, G. C. de, Sassi, F. C., Zambom, L. S., \& Andrade, C. R. F. de. (2016). Correlação entre a gravidade de pacientes críticos e preditores clínicos de risco para a broncoaspiração. J Bras Pneumol, 2(42), 114-120. https://doi.org/10.1590/S1806-37562015000000192

Nascimento, N. P. G. do, Gonçalves, P. F., Douglas-de-Oliveira, W. D., \& Flecha, O. D. (2018). Aspiration Pneumonia and oral health: a critical review of literature. Revista Brasileira de Odontologia, 75, 1-7.

Oliveira, A. C. M. de, Friche, A. A. de L., Salomão, M. S., Bougo, G. C., \& Vicente, L. C. C. (2018). Predictive factors for oropharyngeal dysphagia after prolonged orotracheal intubation. Brazilian Journal of Otorhinolaryngology, 84(6), 722-728. https://doi.org/10.1016/j.bjorl.2017.08.010 
Research, Society and Development, v. 11, n. 1, e27111124847, 2022

(CC BY 4.0) | ISSN 2525-3409 | DOI: http://dx.doi.org/10.33448/rsd-v11i1.24847

Paine, M. A. (2017). Dysphagia: A New Geriatric Syndrome. Journal of the American Medical Directors Association. 18(7):555-557. doi: 10.1016/j.jamda.2017.03.017.

Pereira, A.S., Shitsuka D.M., Parreira, F.J., Shitsuka, R. Metodologia da pesquisa cietífica. 1ªed. (2018). Santa Maria/RS.

Porto, A. L., \& Porto, C. C. (2019). Semiologia médica. Guanabara Koogan.

Schrock, J. W., Bernstein, J., Glasenapp, M., Drogell, K., \& Hanna, J. (2011). A novel emergency department dysphagia screen for patients presenting with acute stroke. Academic Emergency Medicine, 18(6), 584-589. https://doi.org/10.1111/j.1553-2712.2011.01087.x

See, K. C., Peng, S. Y., Phua, J., Sum, C. L., \& Concepcion, J. (2016). Nurse-performed screening for postextubation dysphagia: A retrospective cohort study in critically ill medical patients. In Critical Care (Vol. 20, Issue 1). https://doi.org/10.1186/s13054-016-1507-y

Toh Yoon, E. W. (2018). A novel semi-solidifying liquid formula via the nasogastric route to maintain enteral nutrition in the event of recurrent aspiration pneumonia: A case report. Clinical Case Reports, 6(9), 1708-1712. https://doi.org/10.1002/ccr3.1668

Weinhardt, J., Hazelett, S., Barrett, D., Lada, R., Enos, T., \& Keleman, R. (2008). Accuracy of a bedside dysphagia screening: A comparison of registered nurses and speech therapists. Rehabilitation Nursing, 33(6), 247-252. https://doi.org/10.1002/j.2048-7940.2008.tb00236.x

Yeo, H., \& Rosenthal, R. (2018). Cirurgia no paciente geriátrico (S. T. de Cirurgia (Ed. 20ª edição). Elsevier Editora Ltda,. 